\title{
Manual lymphatic drainage improves the quality of life in patients with chronic venous disease: a randomized controlled trial
}

\author{
Pawel Molski ${ }^{1,2}$, Jacek Kruczyński ${ }^{2}$, Andrzej Molski ${ }^{3}$, Stanislaw Molski
}

1Department of Physiotherapy, Eskulap Health Centre, Bydgoszcz, Poland 2Department of General Orthopaedics, Musculoskeletal Oncology and Trauma Surgery, Poznan University of Medical Sciences, Poznan, Poland

${ }^{3}$ Department of Vascular Surgery, Eskulap Health Centre, Bydgoszcz, Poland

\section{Submitted: 7 July 2011}

Accepted: 2 October 2011

Arch Med Sci 2013; 9, 3: 452-458

DOI: 10.5114 /aoms.2013.35343

Copyright $\odot 2013$ Termedia \& Banach

\author{
Corresponding author: \\ Pawel Molski PT, PhD \\ Department of Physiotherapy \\ Eskulap Health Centre \\ 41 Cicha St \\ 85-650 Bydgoszcz, Poland \\ Phone: +48502021260 \\ Fax: +48 (52) 3602356 \\ E-mail: \\ p.molski@szpitaleskulap.pl
}

\begin{abstract}
Introduction: Manual lymphatic drainage (MLD) is an adjunctive method of chronic venous disease (CVD) therapy. Evaluation of the change at the clinical stage, hemodynamic parameters and quality of life $(\mathrm{Q} O \mathrm{~L})$ following venous system surgery in CVD patients undergoing MLD preoperatively are the most interesting aspects of the study.

Material and methods: The CVD patients qualified for elective surgery of the venous system were randomly divided into 2 groups: the MLD group $(n=38)$ and the control group $(n=32)$. In the preoperative period, the MLD group underwent a series of MLD through a period of 2 weeks. The control group did not undergo MLD preoperatively. Both groups were evaluated for CVD staging on the day of qualification for surgery and between 25 and 30 days post-operatively. Additionally, the MLD group was evaluated after the series of MLD. The CVD staging was evaluated in both groups with a QoL questionnaire (CIVIQ), CEAP classification, foot volumetry (FV) and venous refilling time (VRT).

Results: Parameter values obtained in the MLD group (before treatment/after MLD/after surgery): CEAP 2.23/2.15/2.10, VRT 15/13/15.6, FV 3625/3472/3418, CIVIQ-complaints: 54.4/43.8/38.2 and CIVIQ-meaning: 57.3/49.3/43.1. Parameter values obtained in control group (before surgery/after surgery): CEAP 2.4/2.12, VRT 13/14.9, FV 3581/3559, CIVIQ-complaints: 51.9/38.7 and CIVIQ-meaning: 53.7/40.6. The CVD patients statistically improved in CEAP staging, FV and QoL in both groups $(p<0.05)$.

Conclusions: The MLD alone significantly reduced FV in patients with CVD, also improving their QoL. The MLD applied in CVD patients at the preoperative stage results in better surgical outcome, which is demonstrated by reduced disease progression, FV reduction and improvement in the QoL.
\end{abstract}

Key words: manual lymphatic drainage, chronic venous disease, quality of life.

\section{Introduction}

Chronic venous disease (CVD) is a condition that leads to significant somatic symptoms, which may cause psychological disorders and difficulties in the field of social activity. Until recently, research on the effects of treatment in patients with CVD focused mainly on the functional condition and the influence of physiotherapy on the level of physical fitness. Intensive development of studies on quality of life $(\mathrm{Q} o \mathrm{~L})$ in medical sciences provided an opportunity to evaluate the QoL in pa- 
tients with CVD. It seems to be a useful diagnostic method, which contributes to the evaluation of disease severity, as well as to evaluation of treatment effects [1-4].

One of the methods of conservative treatment of CVD is manual lymphatic drainage (MLD). However, this method is rarely used in patients with venous insufficiency. Therefore, it seems to be justified to commence research on the use of MLD in patients with CVD. The aim of the study was to evaluate the influence of MLD on clinical staging, QoL and hemodynamic parameters in CVD patients, as well as changes in clinical manifestation, hemodynamic parameters and QoL after varicose vein surgery in patients who underwent a series of MLD before surgery.

\section{Material and methods}

The study included 216 consecutive patients with a diagnosis of CVD, qualified for elective surgery and compression therapy for this reason. The study was conducted in the Eskulap Phlebology Centre in Bydgoszcz. Inclusion criteria were CEAP stage C2 and C3, patient willing to undergo elective stripping of the great saphenous vein (GSV), and consent for the study. Exclusion criteria: not willing to undergo surgery, incompetence of deep venous system described as a reflux longer than $0.5 \mathrm{~s}$, chronic lower limb ischemia defined as a lack of distal pulses or signs of ambulatory claudication Fontaine grade II to IV, skin infection, severe comorbidities described as ASA grade III to $\mathrm{V}$, presence of neoplastic disease, lymphangitis, heart failure and renal failure. The study included 80 patients aged from 25 to 65, 70 women (average age 50 years) and 10 men (average age 49 years) with CVD stage CEAP C2 or C3, who were randomly divided into two groups: the MLD group $(n=38)$ and the control group $(n=32)$. The randomization was conducted using 40 sealed envelopes which directed patients to the MLD group and 40 envelopes that directed patients to the control group. In order to randomize a patient's choice of one of the remaining envelopes, open envelopes were discarded. Two patients from the MLD group and 8 from the control group were lost to follow-up and excluded from the study. The study was performed according to the study's algorithm (Figure 1).

Qualification for elective GSV stripping due to CVD was based on clinical evaluation and lower extremities venous system ultrasound duplex examination performed by a vascular surgeon with 30 years' experience. The qualification criteria for elective GSV stripping were clinical signs of CVD and presence of GSV reflux longer than $0.5 \mathrm{~s}$ following a Valsalva maneuver or distal vein compression.
The study group was made up of 38 patients with CVD, qualified for surgery, who had preoperatively undergone a series of MLD.

The MLD included the CVD limb affected and it was performed according to the technique of Vodder [5]. Each person in the study group underwent a series of $10 \mathrm{MLD}$. Each procedure was performed 5 times a week for 20 mins. The control group included 42 patients with CVD, who had been qualified for surgery without undergoing the series of MLD.

All randomized patients underwent the following: clinical evaluation according to CEAP classification complemented with scale of pathological changes severity, photoplethysmography, foot volumetry, QOL examination according to civiq questionnaire.

One day after the last series of MLD, the patients from the study group underwent: clinical examination according to CEAP classification complemented with scale of pathological changes severity, photoplethysmography, foot volumetry, QoL examination according to CIVIQ questionnaire.

All patients included in the control group, as well as those in the study group, underwent elective venous surgery due to CVD and were recommended to use $2^{\text {nd }}$ degree compression therapy.

Surgery in both groups started from venous system mapping by duplex-Doppler USG. First, perforating and deep system competence and patency were evaluated. Next, main superficial trunks incompetence and level of venous reflux were evaluated. Surgery of the incompetent GSV was started in the groin with a skin incision located in the inguinal crease. The sapheno-femoral junction (SFJ) was dissected. The GSV was ligated and cut as close as possible to the common femoral vein (CFV) but without leaving a CFV stricture. All collateral veins of the SFJ were ligated and cut. The sapheno-popliteal junction was dissected at the level marked during USG mapping. The small saphenous vein (SSV) was ligated in the place where it pierced the muscular fascia in order to join the popliteal vein. The distal part of the GSV or SSV was stripped in the whole incompetent part with a Babcock stripper. Any major varicose collaterals were removed by mini-phlebectomy with use of an Oesch hook. Skin wounds were sutured and sterile dressing and compression bandages were applied. All patients were discharged the next day post-operatively and scheduled for a follow-up visit after 7 days. Patients received the recommendation to use compression therapy and oral pain killer medication (ketoprofen $3 \times 100 \mathrm{mg}$ ). During post-operative visits patients were evaluated for possible adverse events. Except for mild thigh bruising and ten- 


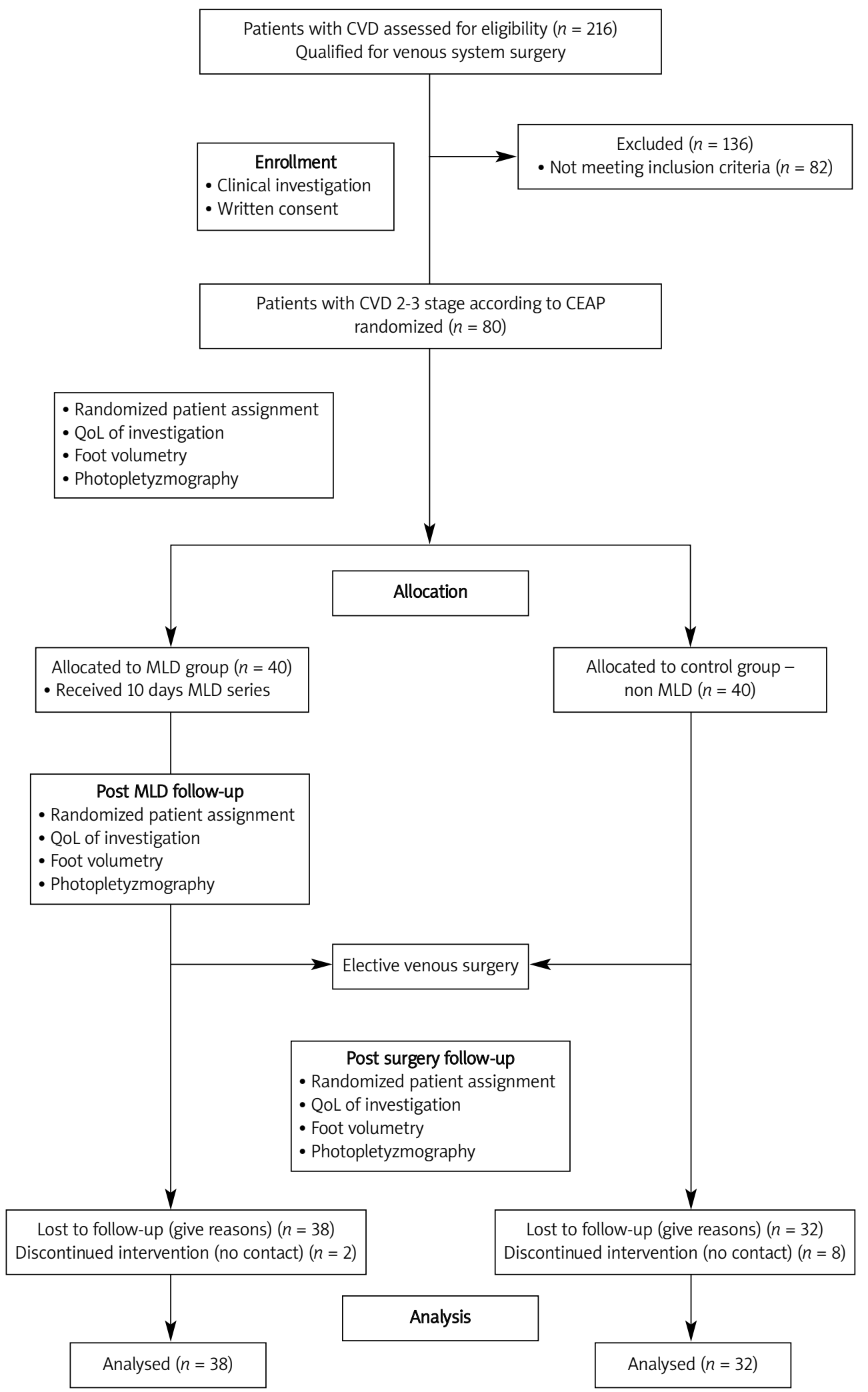

Figure 1. The algorithm of the research process

derness, no adverse events were observed during the post-operative period.

One month after the surgery, the patients underwent a follow-up examination, which consisted of: cli- nical examination according to the CEAP classification complemented with a scale of pathological changes severity, photoplethysmography, foot volumetry, QoL examination according to CIVIQ questionnaire. 


\section{Data analysis}

At the first stage of statistical proceedings, the Shapiro-Wilk test was performed to determine whether the distribution of the studied quantitative parameters (venous refilling time, foot volumetry and QoL) followed a normal distribution. As the distribution of the parameters did not follow a normal distribution, later on in the course of statistical procedures, nonparametric tests were applied, i.e. Mann-Whitney $U$-test and Wilcoxon matched pairs test. The first test was used to compare the control group with the study group in terms of foot volumetry and QoL, whereas the Wilcoxon matched pairs test was carried out to evaluate the significance of differences between the values of particular measurements obtained on the day of qualification and after MLD was carried out. The statistical influence of the group and of the administered therapy on ordinal features was examined by means of the $\chi^{2}$ test for independence.

\section{Results}

\section{Clinical disease severity measurement}

On the day of qualification, the average degree of severity in the study group according to CEAP was 2.23. After MLD the average value of the clinical CEAP score decreased to $2.15(p<0.05)$. In the MLD group after surgery the average value of CEAP decreased to $2.10(p<0.05)$. The clinical picture improved also in the control group, where the CEAP score decreased from 2.40 to 2.12 ( $p<0.05)$. After surgery no statistically significant differences between the MLD group and the control group were observed (Figure 1).

\section{Pain intensity measurement}

In the MLD group, after a series of MLD, pain symptoms decreased from 0.95 to $0.08(p<0.05)$.
The intensity of pain decreased in both groups in the course of the experiment (Table I). After surgery no statistically significant differences between the MLD group and the control group were observed.

\section{Edema intensity measurement}

In the MLD group after a series of MLD, a delay in occurrence of edema during the day was observed $(p<0.05)$. Also in both groups edema was observed later regarding the time of day $(p<0.05)$. No statistically significant differences between the MLD group and the control group were observed (Table II).

\section{Quality of life measurement}

After a series of MLD, in the MLD group the QoL improved regarding the symptoms, which decreased according to a point-based scale by $19.5 \%$ from 54.4 to 43.8 ( $p<0.05)$. After surgery in the MLD group the average of symptoms further decreased by $12.8 \%$ to 38.2 ( $p<0.05$ ). In the control group it decreased from 51.9 to $38.7(p<0.05)$. No statistically significant differences between the MLD group and the control group were observed (Figure 3). In both groups the subjective evaluation of degree of symptoms experienced by patients decreased $(p<0.05)$.

In the MLD group the average degree of symptoms experienced after MLD decreased by $14 \%$ from 57.3 to 49.3 . After surgery it further decreased by $12.6 \%$ to 43.1 . In the control group, the average degree of symptoms experienced after surgery decreased by $24.4 \%$ from 53.7 to 40.6 . No statistically significant differences between the MLD group and the control group were observed (Figure 3).

\section{Venous refilling time measurement}

In both groups no statistically significant differences were observed after surgery (Figure 4).

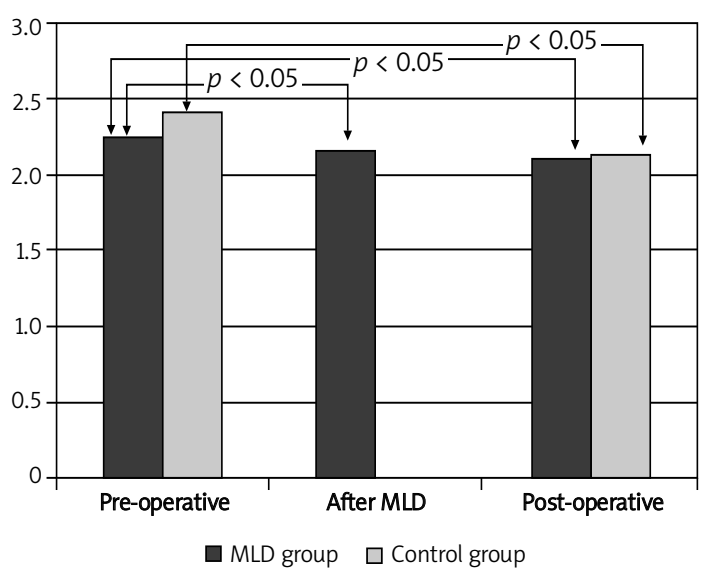

Figure 2. Comparison of average values of clinical stage according to CEAP classification

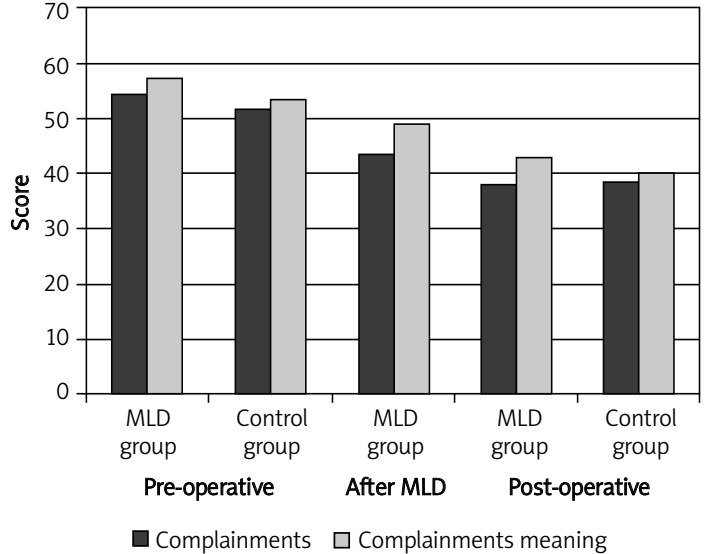

Figure 3. Comparison of average values of symptoms and their significance 
Table I. Pain intensity in control and MLD group

\begin{tabular}{|lccccc|}
\hline \multicolumn{1}{|c|}{ 0-No } & 1-Moderate & 2-Strong & 3-Very strong & Total \\
\hline MLD group & & & & \\
\hline On the day of qualification & $7(18.42 \%)$ & $27(71.05 \%)$ & $3(7.89 \%)$ & $1(2.63 \%)$ & 38 \\
\hline After surgery & $33(86.84 \%)$ & $5(13.16 \%)$ & $0(0 \%)$ & $0(0 \%)$ & 38 \\
\hline Control group & & & & \\
\hline On the day of qualification & $4(12.50 \%)$ & $23(71.88 \%)$ & $5(15.63 \%)$ & $0(0 \%)$ & 32 \\
\hline After surgery & $29(90.63 \%)$ & $3(9.38 \%)$ & $0(0 \%)$ & $0(0 \%)$ & 32 \\
\hline
\end{tabular}

Table II. Comparison of time of occurrence of edema regarding the time of day

\begin{tabular}{|lccccc|}
\hline \multicolumn{1}{|c|}{ 0-No } & 1-Evening & 2-Afternoon & 3-Morning & Total \\
\hline MLD group & & & & \\
\hline On the day of qualification & $11(28.95 \%)$ & $16(42.11 \%)$ & $7(18.42 \%)$ & $4(10.53 \%)$ & 38 \\
\hline After surgery & $32(84.21 \%)$ & $5(13.16 \%)$ & $1(2.63 \%)$ & $0(0 \%)$ & 38 \\
\hline Control group & & & & \\
\hline On the day of qualification & $8(25.00 \%)$ & $12(37.50 \%)$ & $10(31.25 \%)$ & $2(6.25 \%)$ & 32 \\
\hline After surgery & $25(78.13 \%)$ & $4(12.50 \%)$ & $2(6.25 \%)$ & $1(3.13 \%)$ & 32 \\
\hline
\end{tabular}

\section{Foot volumetry (FV) measurement}

In the MLD group after a series of MLD the average of FV decreased from $3625 \mathrm{ml}$ to $3472 \mathrm{ml}$ $(p<0.05)$ and after surgery it was $3418 \mathrm{ml}$. In the control group after surgery the average of $\mathrm{FV}$ decreased from $3581 \mathrm{ml}$ to $3559 \mathrm{ml}$ ( $p<0.05$ ). In the MLD group the improvement was higher $(p<0.01)$ than in the control group (Figure 5).

\section{Discussion}

In 2008, the guidelines of the expert group headed by Nicolaides concerning classification, diagnostics and treatment of CVD were published. Evaluation of QoL was presented as an important tool in the analysis of treatment results. QoL questionnaires, such as CIVIQ, VCSS, SF-36, and $\mathrm{HAD}$, were considered as very useful and credible in evaluation of disease severity and in evalua-

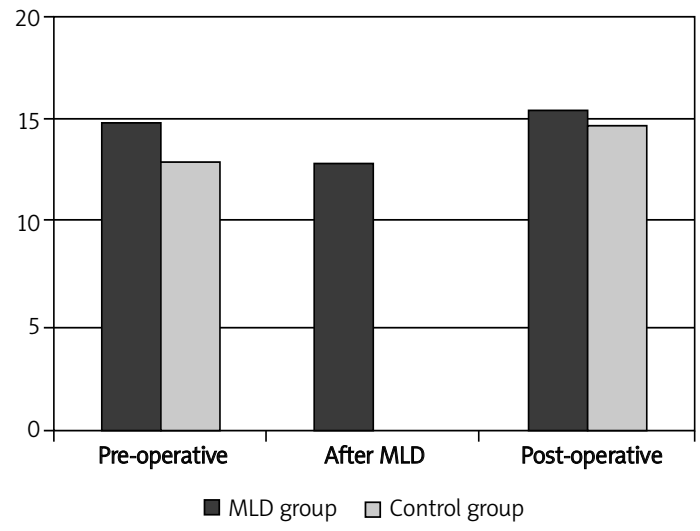

Figure 4. Comparison of average VRT values tion of conservative treatment as well as surgical treatment [6].

In conservative treatment of CVD methods such as elimination of risk factors, pharmacotherapy, compression therapy or physical exercise have a well-proven track record, whereas MLD has not achieved the position it deserves yet. There are not many publications concerning the role of MLD in the treatment of CVD.

Research has proven that MLD applied in patients with CVD improves clinical stage, hemodynamic parameters and QoL. In evaluation of disease severity in patients with CVD, significant improvement of clinical stage was observed after MLD. The average value of clinical stage according to CEAP improved by $3.6 \%$. We observed significant improvement of lower limb and foot volumes. After 10 days of MLD the volumes of foot and lower limb decreased by $153 \mathrm{ml}(p<0.05)$ on average.

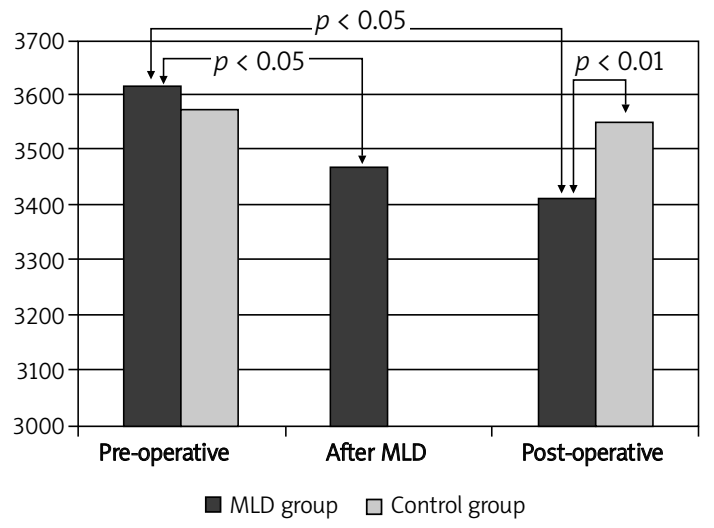

Figure 5. Comparison of average FV values 
The mean of the QoL parameter regarding symptoms decreased by $19.5 \%$, and the mean of the QoL parameter regarding the significance of symptoms decreased by $14 \%$.

In earlier studies it was proven that after performance of MLD in patients with CVD, the average of clinical parameter $C$ according to the CEAP classification improved from 3.6 to 1.5 . Using the HAD questionnaire, we proved that CVD causes anxiety and depressive states, and MLD improved QoL by decreasing these symptoms. The average of anxiety states after MLD decreased from 12.8 to 4.9. The average of depressive states also decreased, from 9.4 to 3.0 [7].

Felty et al. emphasized that compression therapy has been used in the treatment of CVD for over 2000 years. In order to improve the effectiveness of this method, he used intermittent compression and MLD. The connection of these two methods caused a decrease of edema [8].

A beneficial influence of MLD on the effects of treatment in patients with CVD was observed by Evrard-Bras et al. They proved that skin care, banding according to the rule of gradual compression and appropriate physical exercise prolong the effects of MLD. They claim that the main indications for MLD are: CVD, primary and secondary lymphatic edema, and post-injury conditions. However, it seems controversial to recommend MLD in thromboembolic disease [9].

Foldi reported the ineffectiveness of MLD without compression therapy in their studies [10]. Grądalski and Ochałek claim that it is essential to simultaneously perform compression therapy in order to prolong the anti-edema effects of MLD [11].

Rowland examined the use of pneumatic compression as an alternative to compression bandage in patients with venous ulcer. Patients were divided into 2 groups. As the pressure of the pneumatic compression device is constant, reduction of edema was equal in both groups during the period when edema was greatest; however, in the progressive aspect, a greater reduction in edema volume was observed in the bandage group [12].

A meta-analysis study prepared by Karki et al. also showed that MLD alone was not effective [13]. Szewczyk et al. proved the effectiveness of MLD in decreasing edema of lower limbs in patients with CVD. In a group of 15 patients, who had MLD performed twice a week for a period of 3 weeks, they evaluated the results of treatment on the basis of lower limbs perimeter measurement. They observed a decrease of edema compared to the initial condition on average by $2.2 \mathrm{~cm}$ around the ankles and $1.7 \mathrm{~cm}$ around the calf [14].

The MLD is an easy therapeutic method but it requires a high level of technical ability to be applied successfully. In this way, this method gives really good results especially in congenital and acquired lymphedema, CVD, and some general diseases such as headaches and colitis [15].

Despite a relatively small number of patients, this study proves that application of MLD before surgery of varicose veins improves the effect of the surgical treatment. Comparative analysis in the studied groups showed better results as regards the disease severity, as well as hemodynamic parameters (foot volumetry) and QoL. As regards the point-based value of disease severity, more significant improvement was observed in the MLD group than in the control group. However, this difference was not statistically significant. There was a statistically significant difference between both groups in foot volumetry measurement.

Our earlier studies proved that there were better effects of surgical treatment, as far as decrease of anxiety and depressive states caused by varicose veins are concerned, in a group of patients who underwent MLD before surgery. The average of anxiety states, as well as the average of depressive states, decreased more significantly in the MLD group than in the control group. These results were positively correlated with the results obtained in clinical examination and hemodynamic test (VRI) [7].

Bakar et al. performed a study in 62 patients, aged 59 to 68 years (mean $64.95 \pm 2.54$ years), with chronic venous disease treated with complex decongestive physiotherapy (CDP). The study results proved that CDP can statistically significantly reduce the volume and percentage volume of CVI-related edema and reduce the intensity of pain caused by this condition in older people. According to the authors, further controlled studies should include a greater number of cases, subjective and objective comparison of MLD outcomes, intermittent pneumatic compression, exercise, the use of compression alone, and comparison with the gold standard for CDP in terms of both short- and long-term results, using objective signs such as pre- and post-treatment Doppler ultrasound results [16].

The surgical treatment of CVD in the lower limbs, whether it is a case of so-called 'essential' varices or of post-phlebitic disorders, presents a certain number of risks of venous thrombosis and especially of cutaneous necrosis or of cicatricial detachment in operations of the Lindon or Felder type. Given a less significant set-back, it has been suggested that restorative surgery of the deep venous network, valve repair or insertion of a valvulated section should be used in the treatment of postphlebitic illness. Promising results have been obtained through specific kinesitherapy, involving a Leduc type MLD together with pressotherapy, in the preparation of patients and in post-operative follow-up care [17]. 
The results obtained in this study on the use of MLD in treatment of patients with CVD are comparable with the results obtained by other researchers $[7,14]$. The beneficial influence of MLD on the improvement of clinical and hemodynamic parameters and on the improvement of QoL has been confirmed in patients undergoing conservative treatment as well as in those who underwent varicose vein surgery. This allows us to assume that MLD is an effective method complementing the methods of CVD treatment that have been used until now.

In conclusion, manual lymphatic drainage decreases the degree of disease severity and foot and lower limb volume in patients with chronic venous disease, as well as improving their quality of life. Manual lymphatic drainage applied in patients with chronic venous disease pre-operatively provides better effects of treatment, which is manifested by a decrease in the degree of disease severity, foot and lower limb volume, and improvement of quality of life.

\section{References}

1. Gloviczki P, Comerota AJ, Dalsing MC, et al. The care of patients with varicose veins and associated chronic venous diseases: clinical practice guidelines of the Society for Vascular Surgery and the American Venous Forum. J Vasc Surg 2011; 53 (5 Suppl): 2S-48S.

2. Biemans AA, van der Velden SK, Bruijninckx CM, Buth J, Nijsten T. Validation of the chronic venous insufficiency quality of life questionnaire in dutch patients treated for varicose veins. Eur J Vasc Endovasc Surg 2011; 42: 246-53.

3. Jawień A, Szewczyk MT, Kędziora-Kornatowska K, et al. Functional and biopsychosocial restrictions among patients with a venous ulcer. Arch Med Sci 2006; 2: 36-41.

4. Bączyk G, Talarska D, Zawirska A, et al. Functioning and quality of life of patients with leg ulcers treated at dermatology wards. Postep Derm Alergol 2011; 28: 191-6.

5. Földi M, Strößenreuther, R. Treatment of the leg. Foundations of manual lymph drainage. 3 ed. Elsevier Mosby 2005.

6. Nicolaides AN, Allegra C, Bergan J, et al. Management of chronic venous disorders of the lower limbs: guidelines according to scientific evidence. Int Angiol 2008; 27: 1-59.

7. Molski P, Ossowski R, Hagner W, Molski S. Patients with venous disease benefit from manual lymphatic drainage. Int Angiol 2009; 28: 151-5.

8. Felty CL, Rooke TW. Compression therapy for chronic venous insufficiency. Semin Vasc Surg 2005; 18: 36-40.

9. Evrard-Bras M, Coupe M, Laroche JP, et al. Manual lymphatic drainage. Rev Prat 2000; 50: 1199-203.

10. Foldi E. The treatment of lymphedema. Cancer 1998; 83 : 2833-4.

11. Ochałek K, Grądalski T. Manual Lymph Drainage usage in vascular diseases. Acta Angiologica 2011; 3: 1-10.

12. Rowland J. Intermittent pump versus compression bandages in the treatment of venous leg ulcers. Aust N Z J Surg 2000; 70: 110-3.

13. Karki A, Anttila H, Tasmuth T, Rautakorpi UM. Lymphoedema therapy in breast cancer patients: a systematic review on effectiveness and a survey of current practices and costs in Finland. Acta Oncol 2009; 48: 850-9.

14. Szewczyk T, et al. The importance of massage in edema of lower limbs in patients with chronic venous insufficiency. Valetudinaria. Postępy Medycyny Klinicznej i Wojskowej 2002; 7: 50-3.

15. Traissac B, Sagardoy G, Lucas JF. Manual lymphatic drainage in angiology. Phlebologie 1988; 41: 471-6.

16. Bakar Y, Ozturk A, Calisal M, Erturk K, Daglar B. Complete decongestive physiotherapy for older people with chronic venous insufficiency. Topics in Geriatric Rehabilitation 2010; 26: 164-70.

17. Valentin J, Léonhardt D, Perrin M. Prevention of venous thromboses and cutaneous necroses using physical methods and pressure therapy in the surgery of chronic venous insufficiency of the lower limbs. Phlebologie 1988; 41: 690-6. 